\title{
THE $H-\Omega_{O}$ DIAGRAM FROM RECENT CMB OBSERVATIONS
}

\author{
YES, WE CAN ALREADY SAY SOMETHING
}

\author{
C.H. LINEWEAVER \\ Observatoire de Strasbourg \\ Strasbourg, France \\ and \\ School of Physics, UNSW \\ Sydney, Australia \\ charley@edwin.phys.unsw.edu.au
}

\begin{abstract}
.
The CMB is already one of the pillars of the Big Bang model. However it may also become our most powerful tool to distinguish contending models and to determine their cosmological parameters. To realize this goal, more than 20 observational groups and two new satellites are gearing up to make precise measurements of the CMB at small angular scales. In such a situation it is important to keep track of what the CMB data can already say about cosmological parameters.

Current CMB data can already be used to constrain cosmological parameters. The results are model dependent. We have obtained contraints on Hubble's constant $h$ and the density of the Universe $\Omega_{o}$ in the context of open and critical density CDM models with $\Lambda=0$. In critical density models we obtain $h=0.30_{-0.07}^{+0.18}$. This low value is inconsistent with direct measurements of $h$ but fully consistent with four other cosmological measurements: Big Bang nucleosynthesis, cluster baryonic fraction, age constraints from globular clusters and limits on the shape parameter $\Gamma$ of matter power spectra (in $\Omega_{o}=1$ models). If $\Omega_{0}$ is left as a free parameter the constraints on $h$ are less restrictive: $h=0.40_{-0.14}^{+0.57}$. This is fully consistent with local $h$ measurements and the four other cosmological measurements mentioned above. The best-fit density is $\Omega_{O}=0.85$ and we set an upper limit of $\Omega_{o}>0.4$ at $\sim 95 \%$ CL.

At this conference Ostriker has claimed that open-CDM models with $\Omega_{o} \approx 0.3$ and $h \approx 0.70$ are compatible with all current data. However our new $\mathrm{CMB}$ data analysis rules this model out at more than $\sim 4 \sigma$.
\end{abstract}




\section{Introduction}

At this conference it has been said that determinations of cosmological parameters from $\mathrm{CMB}$ data is something that will be done in the future-that the scatter in the data does not allow one to conclude anything yet. However the results presented here show that current CMB data can already rule out large regions of parameter space under certain popular assumptions, e.g., adiabatic initial conditions, no tensor modes, $\Lambda=0$. The details of these assumptions as well as results for the slope $n$ and amplitude $Q$ of the primordial power spectrum and the position and amplitude of the peak in the power spectrum are given in Lineweaver \& Barbosa (1997a \& b). Here I limit the discussion to the CMB constraints on $h$ and $\Omega_{o}$.

Figure 1 is a compilation of current CMB data. Although there is much scatter, simple binning of the data brings out a very significant peak at $\ell \sim 260$. We do $\chi^{2}$ fits of model power spectra to this data. The power spectra are calculated with a fast Boltzmann code (Seljak \& Zaldarriaga, 1996). Our results are displayed in Figures 2 and 4 where the $\Delta \chi^{2}$ contours are for $\Delta \chi^{2}=[1,4,9]$ corresponding to $[68 \%, 95 \%, 99 \%]$ confidence regions.

\section{Figure 1.}

Current CMB Data The

solid line is the best-fitting model in the open and critical density family of models. The $\chi^{2}$ fit is good. The grey band is the variation of these models permitted within the $68 \%$ confidence region of Figure 4. Experiments designed optimally to constrain parameters should have window functions in regions where the vertical spread of the grey band is largest. Figure from Lineweaver \& Barbosa (1997b).

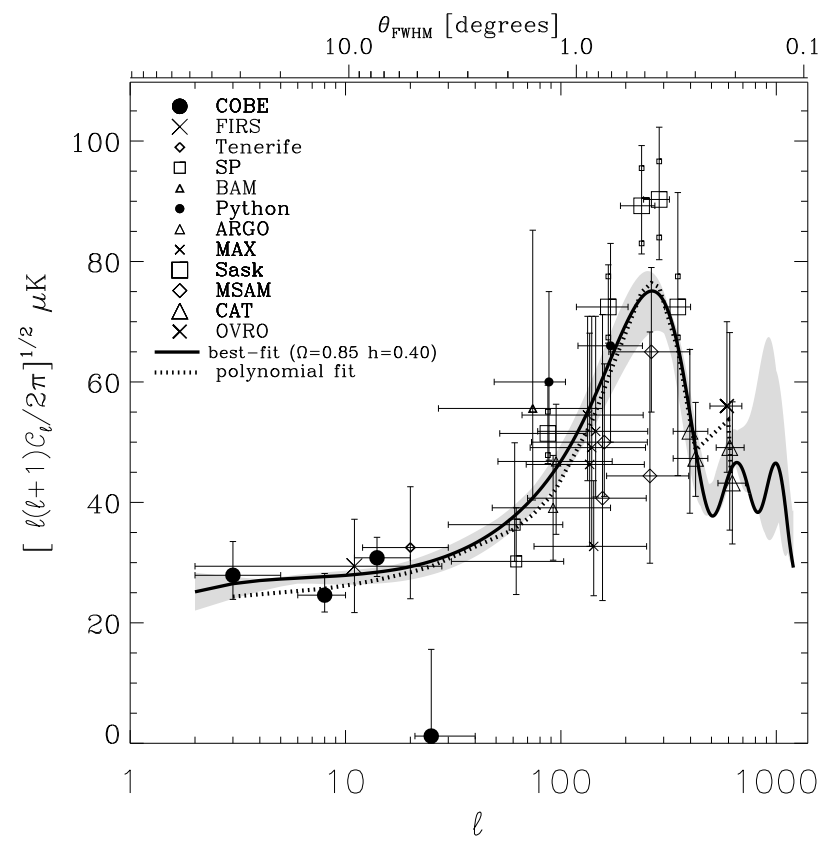


Figure 2.

CMB Constraints in the $h-\Omega_{b}$ plane when $\Omega_{o}=$ 1. The dark grey area is the approximate $68 \%$ confidence region about the best-fit at $h=0.30_{-0.07}^{+0.18}$ (marked with a $\mathbf{X}$ ). The light grey band is from big bang nucleosynthesis $\left(0.010<\Omega_{b} h^{2}<0.026\right)$. Figure from Lineweaver \& Barbosa (1997a).

Figure 3.

Non-CMB constraints in the $h-\Omega_{b}$ plane when $\Omega_{o}=$ 1 The light grey band is from big bang nucleosynthesis and is the same in Figure 2. The two other bands are the preferred regions from baryonic fractions in clusters and the matter power spectrum $(\Gamma)$. The vertical lines indicate the range for the age of the Universe inferred from the oldest stars in globular clusters. An approximate joint likelihood of these constraints yields the dark grey $68 \%$ confidence region: $h=0.40 \pm 0.07$. Figure from Lineweaver \& Barbosa (1997a).
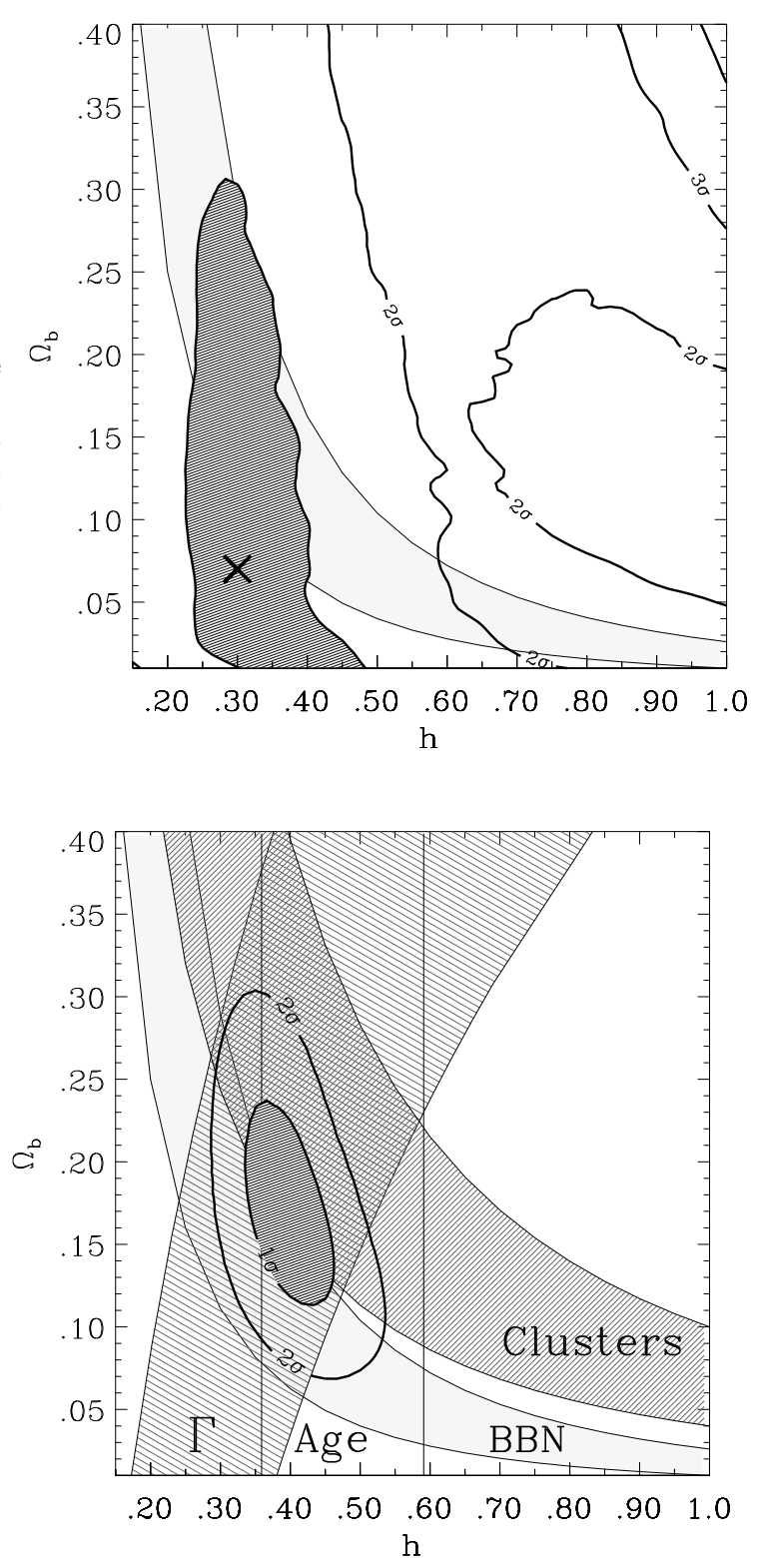

By comparing Figures 2 and 3 we see that the CMB and Non-CMB constraints are consistent in the sense that the $68 \%$ confidence regions overlap. However the overlap is at $h \approx 0.35$. This is inconsistent with local measurements of Hubble's constant which favor $h \approx 0.65 \pm 0.15$. The assumption that $\Omega_{o}=1$ has led to this inconsistency. Figures 4 and 5 show that this inconsistency is removed when we let $\Omega_{o} \leq 1$. 
Figure 4.

CMB constraints in the $h-$ $\Omega_{o}$ plane. The dark grey region is the $68 \%$ confidence region around the best-fit at $h=$ $0.40_{-0.14}^{+0.57}$ (marked with an $\mathbf{X}$ ). The narrow banana-shaped region means that plausible values of $h$ and $\Omega_{o}$ are correlated and this region defines a new constraint: $\Omega_{o} h^{1 / 2}=0.55 \pm$ 0.10 . The age constraint shown is $10<t_{o}<18$ Gyr. Figure adapted from Lineweaver \& Barbosa (1997b).

Figure 5.

Non-CMB constraints in the $h-\Omega_{o}$ plane. Approximate joint likelihood from the baryonic fraction in clusters $\left(0.10<\Omega_{o} h^{1 / 2}<0.65\right)$, globular cluster ages $\left(10<t_{o}<18\right.$ Gyr), the matter power spectrum $(0.169<\Gamma<0.372)$ and local measurements of Hubble's constant $(0.50<h<$ $0.80)$. Figure from Lineweaver \& Barbosa (1997b).
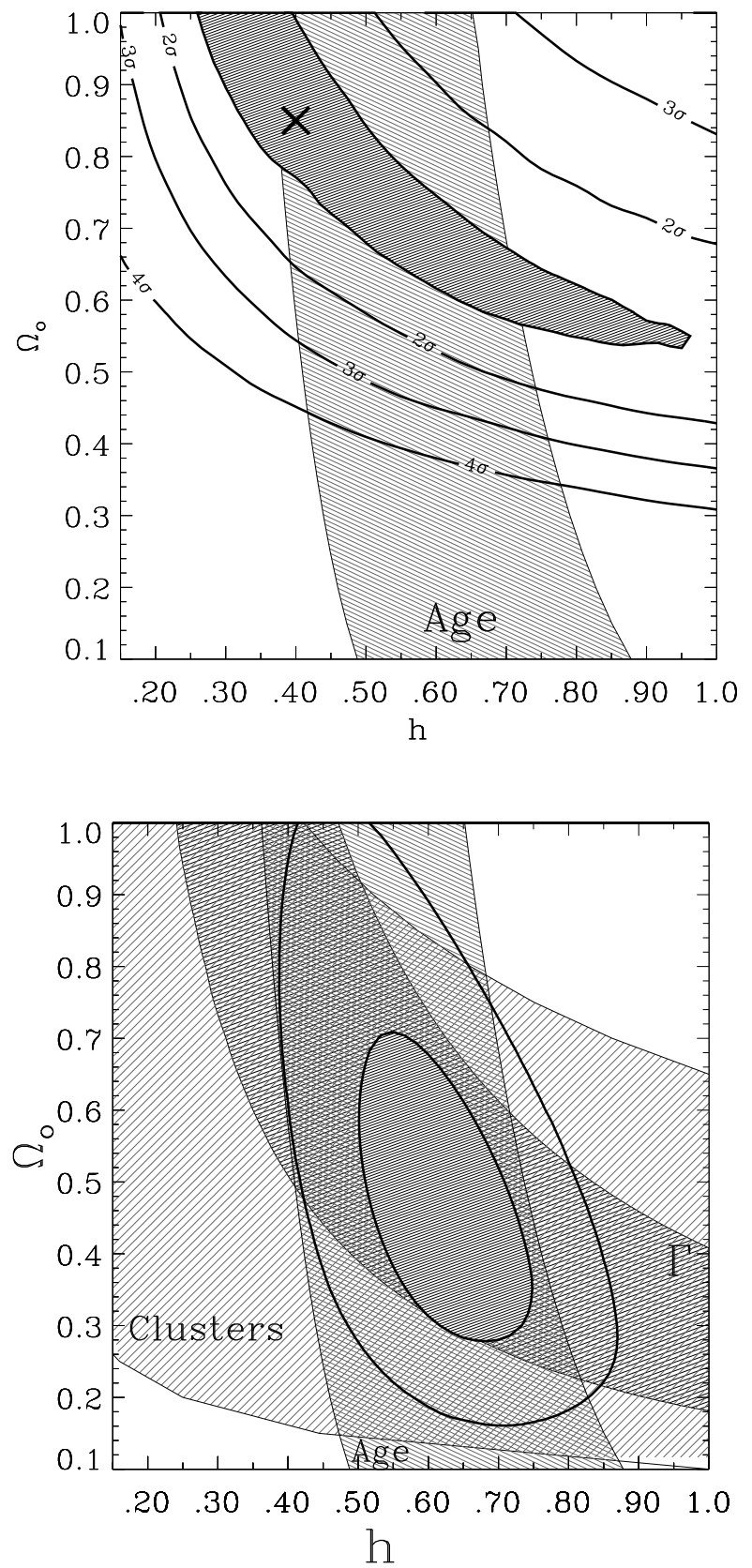

Figure 4 shows that letting $\Omega_{o}$ be a free parameter loosens the constraint on $h$ but that a very narrow banana in the $h-\Omega_{o}$ plane is preferred. This region is consistent with the non-CMB constraints as well as with the local measurements of $h$ since the $68 \%$ confidence regions of Figures 4 
and 5 overlap. An interesting common feature in both plots is that high $\Omega_{0}$ values prefer low $h$ while low $\Omega_{0}$ values prefer high $h$. Notice that the $\Omega_{o} \approx 0.3, h \approx 0.70$ model cited by Ostriker as a model consistent with all observational data is acceptable to the non-CMB data but is ruled out by the CMB data at more than $\sim 4 \sigma$.

Figure 6 combines both $\mathrm{CMB}$ and non-CMB observations. The maximum likelihood is at $h=0.58 \pm 0.11$ and $\Omega_{o}=0.65_{-0.15}^{+0.16}$. Critical density universes, i.e., $\Omega_{o}=1$, are marginally excluded at about the $95 \%$ confidence level. The low density universe mentioned by Ostriker $\left(h \approx 0.70, \Omega_{o} \approx 0.3\right)$ is excluded at slightly more than the $95 \%$ confidence level. My conclusion is that CMB data can already be used as a tool to distinguish contending cosmological models and determine cosmological parameters.

Figure 6.

CMB + non-CMB constraints in the $h-\Omega_{o}$ plane. When the CMB constraint from Figure $4\left(\Omega_{o} h^{1 / 2}=\right.$ $0.55 \pm 0.10)$ is combined with the non-CMB constraints in Figure 5 we obtain $h=0.58 \pm$ 0.11 and $\Omega_{o}=0.65_{-0.15}^{+0.16}$.

Figure from Lineweaver \& Barbosa (1997b).

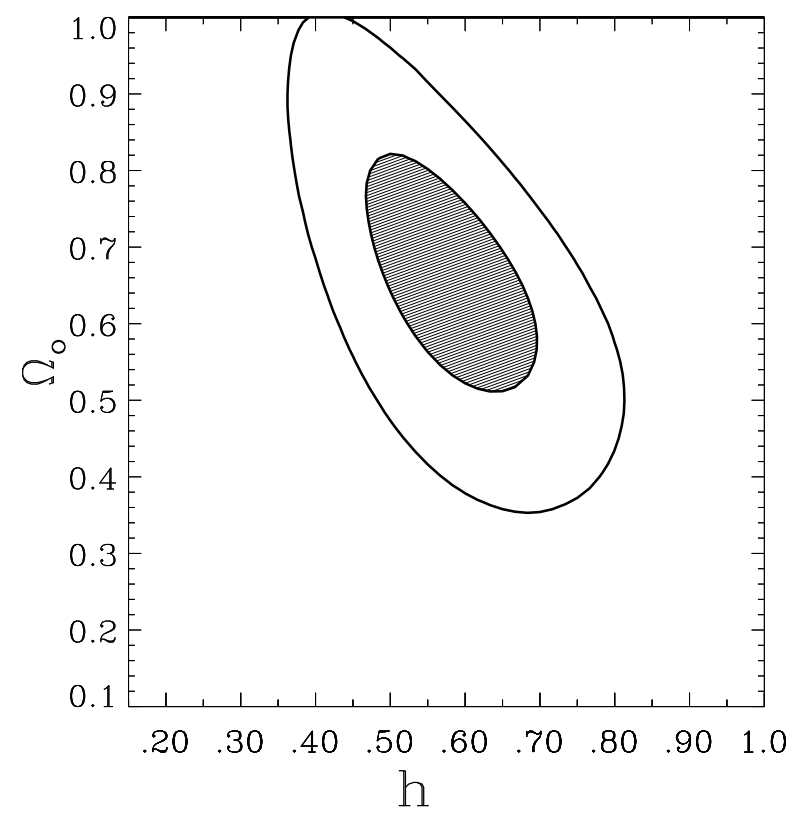

\section{References}

1. Lineweaver, C.H. \& Barbosa, D. (1997a), "Cosmic microwave background observations: implications for Hubble's constant and the spectral parameters $n$ and $Q$ in cold dark matter critical density universes", Astronomy $\& 3$ Astrophysics, in press

2. Lineweaver, C.H. \& Barbosa, D. (1997b), "What Can Cosmic Microwave Background Observations Already Say About Cosmological Parameters in Open and CriticalDensity Cold Dark Matter Models?", Astrophysical Journal, in press

3. Seljak, U. \& Zaldarriaga, M. (1996), "A Line of Sight Approach to Cosmic Microwave Background Anisotropies", Astrophysical Journal, 469, 437 